\title{
Evidence to Support Wearing Masks Is Helpful During the COVID-19 Pandemic
}

\author{
William Tse ${ }^{1}$, MD, Charlotte Tse ${ }^{2}$, MD, Iris Kufferath ${ }^{3}$, and Lukas Kenner ${ }^{4,5,6}$, MD, PhD \\ 1 MetroHealth System, Case Western Reserve University School of Medicine, Cleveland, $\mathrm{OH}$ \\ 2 Clinical trial unit,Cleveland Clinic Foundation, Cleveland, $\mathrm{OH}$ \\ 3 Institute of Pathology, Medizinsche Universität Graz, Graz, Austria \\ 4 Department of Pathology, Medical University Vienna, Vienna, Austria \\ 5 Unit for Laboratory Animal Pathology, University of Veterinary Medicine Vienna, Vienna, Austria \\ 6 Christian Doppler Laboratory for Applied Metabolomics (CDL-AM), Medical University of Vienna.
}

The coronavirus outbreak is primarily a human tragedy, but it is increasingly impacting the global economy. It has created an unprecedented, irrational atmosphere of panic within our society. There is conflicting information about the pandemic - but it is not just about health. We witnessed, for example, how people stockpiled incredible amounts of toilet paper, even though COVID-19 is not a gastrointestinal pathogen.

In contrast to Europe and the US, Singapore, Hong Kong, Taiwan, South Korea, and Japan all kept case numbers relatively low. What have they done differently compared to us?

Besides social distancing, personal hygiene, widespread virus testing, and isolation, the main difference is the wearing of masks in public. This practice is common, especially since the 2003 SARS outbreak. Asian countries have a much higher population density than most Western countries. In Taiwan, the government favours the wearing of masks. Citizens of Hong Kong, South Korea, Japan, and Singapore proactively wear masks in public spaces despite the absence of government policy. Owing to these remarkable practices, these countries have not experienced the same terrible outbreaks as Western countries, and negative effects on the economy have been less pronounced.

As healthcare workers, we urge our scientific communities and governments to rethink the strategy on mask wearing recommendations. As masks effectively reduce the viral load, ${ }^{1}$ and a large percentage of infections is asymptomatic, ${ }^{2}$ wearing them from the beginning of a COVID19-type outbreak is essential. The WHO recommends wearing masks only when an individual develops upper respiratory symptoms, but there is mounting evidence that COVID-19 and flu viruses, also likely airborne, may be contagious before symptoms manifest. Therefore, the WHO's general recommendations should include the wearing of masks. ${ }^{3}$ Moreover, people must be instructed on how to use and dispose of masks correctly. ${ }^{4}$ The fact that the general public in the USA/Europe hardly wears masks might at least partly explain the massive difference in the number of 2019-nCoV positive cases and deaths. ${ }^{5}$

Hence, when comparing the costs of making our citizens wear masks, albeit not too convenient to our lifestyles, to a lock-down of almost the entire economy, the former approach is apparently an effective measure to decelerate the spread, with the goal of 
"flattening the curve". Although wearing masks should never replace or substitute other effective measures recommended by public health agencies and experts, the evidence in Asian countries suggests that it might additionally enhance the efficacy of preventive measures in today's detrimental COVID-19 outbreak.

\section{References}

1 Davies A, Thompson KA, Giri K, Kafatos G, Walker J, Bennett A. Testing the efficacy of homemade masks: would they protect in an influenza pandemic? Disaster Med Public Health Prep. 2013; 7(4):413-8.

2 Lauer SA, Grantz KH, Bi Q, et al. The Incubation Period of Coronavirus Disease 2019 (COVID-19) From Publicly Reported Confirmed Cases: Estimation and Application. Ann Intern Med. 2020; [Epub ahead of print March 10, 2020].

3 World Health Organization

Q\&A on coronaviruses (COVID-19)

https://www.who.int/news-room/q-a-detail/q-a-coronaviruses

Date: March 9, 2020

Date accessed: March 26, 2020

$4 \quad$ World Health Organization

Coronavirus disease (COVID-19) advice for the public: when and how to use masks.

https://www.who.int/emergencies/diseases/novel-coronavirus-2019/advice-for-public/when-andhow-to-use-masks

Date: 2020

Date accessed: March 26, 2020

5 Feng S, Shen C, Xia N, Song W, Fan M, Cowling BJ. Rational use of face masks in the COVID-19 pandemic. The Lancet Respiratory Medicine. 2020; [Published Online March 20, 2020]. 